\title{
HYPOXIC RESPONSE
}

\section{First hints of new sensors}

A mechanism by which plants detect and respond to oxygen starvation has been known for some years. Three recent papers suggest that we haven't been seeing the full picture.

\section{Michael J. Holdsworth}

Low oxygen (hypoxia) is a key abiotic stress experienced by plants during flooding, and so has a major effect on yield in agriculture ${ }^{1}$. However, it is a normal component of plant development ${ }^{2,3}$ and in an ecological context has a major role in patterns of plant distribution and abundance 4 . Three recently published papers reveal new and unexpected facets of the sensing of oxygen by plants. Giuntoli and colleagues ${ }^{5}$ uncover an age-related mechanism controlling the activity of the known plant oxygen sensing proteins, and Eysholdt-Derzsó and Sauter ${ }^{6}$ find a new hypoxia-related root phenotype. One inescapable conclusion from these two studies is that currently unknown sensors of oxygen exist in plants; Wang and coworkers ${ }^{7}$ have used informatics approaches to identify plant proteins that they suggest may fulfil these functions.

A mechanism of oxygen sensing in plants was discovered simultaneously and independently in Arabidopsis thaliana by myself and Francesco Licausi in $2011^{8,9}$. This beautifully simple mechanism involves the sensing of the oxidation status of the cysteine (Cys) residue at the amino-terminus of Group VII ETHYLENE RESPONSE FACTOR (ERFVII) transcription factors by the $\mathrm{N}$-end rule pathway of ubiquitin mediated proteolysis. A hallmark of ERFVII proteins is the initiating 7 residues (in angiosperms with slight variations on the amino acid sequence MCGGAIL-). Constitutive removal of amino-terminal methionine by amino-peptidases reveals Cys-2, which under normal oxygen (and nitric oxide ${ }^{10}$ ) levels is oxidized, allowing the addition of arginine by the $\mathrm{N}$-end rule pathway, subsequent recognition by the $\mathrm{N}$-end rule pathway E3 ligase PROTEOLYSIS 6 (PRT6), and degradation. In the presence of oxygen ERFVII proteins are constitutively degraded, aided by the PLANT CYSTEINE OXIDASE (PCO) enzymes ${ }^{11}$, but as oxygen levels decline they are stabilized, and induce transcription of key genes associated with anaerobic metabolism (for example, ALCOHOL DEHYDROGENASE) $)^{12}$ that enhance survival by providing an alternative, non-oxygen requiring energy generating mechanism ${ }^{13}$.

Giuntoli et al. uncover an age-dependent activity that influences ERFVII function in oxygen sensing and the mitochondrial oxidative stress response in A. thaliana. They show that, in addition to the known fermentation-related genes up-regulated by ERFVIIs, transcripts encoding proteins involved in oxidative responsiveness are also positively controlled by this transcription factor sub-family; this may explain results showing that ectopic expression of ERFVIIs enhances plant survival of many different abiotic stresses (reviewed in ref. 14). $\mathrm{N}$-end rule regulated ERFVII activity was shown to be controlled by plant age, as mature plants displayed distinctly lower induction of ERFVII-regulated genes than young seedlings. The nature of this age-related factor is unknown, though it has previously been shown that ERFVIIs harbour degrons (domains inducing ubiquitin-mediated proteolysis) in addition to the domain required for oxygen sensing that may be activated by light ${ }^{3,15}$. This indicates that degrons regulated by other biochemical or developmental pathways may exist in ERFVII proteins and control their stability. 
In a different approach, Eysholdt-Derzsó and Sauter identify a new and potentially agriculturally important hypoxia-regulated phenotype. They show that hypoxia causes the primary root of $A$. thaliana to become agravitropic and bend upwards. They speculate that this is a response of roots to avoid hypoxic soil environments. Counterintuitively, this response is counteracted by ERFVIIs, whose functions inhibit agravitropic bending, perhaps because ERFVII-induced fermentative metabolism permits survival of hypoxia, allowing plants to explore such soil surroundings. As expected, the flux of auxin in this response is antagonistically regulated by hypoxia and the ERFVII RELATED TO APETALA 2.12 (RAP2.12).

Collective analysis of these studies, based on the experimental evidence provided, suggests that new, non-ERFVII oxygen sensors exist (Fig. 1). In both cases this evidence relies on the use of a pentuple mutant that lacks activity of all five $A$. thaliana ERFVIIs ((rap2.12, rap2.2, rap2.3, hre 1, and hre 2, hereafter referred to as erfVII ${ }^{3}$ ). Lacking the activity of all ERFVII oxygen sensors, the pentuple mutant should be insensitive to hypoxia. Previous work confirmed this by showing that maintenance of the apical hook of skotomorphogenic (dark grown) seedlings under hypoxia was completely abolished in the erfVII mutant ${ }^{3}$. Giuntoli et al. show that, in erfVII seedlings in an anoxic gaseous environment, expression of fermentation-associated genes is abolished because seedlings are unable to sense oxygen and activate downstream gene expression. However, in either erfVII adult plants or seedlings that are both subjected to $24 \mathrm{~h}$ submergence, the expression of both hypoxia and oxidative genes is still up-regulated, highly suggestive of ERFVII-independent low-oxygen sensing. Eysholdt-Derzsó and Sauter show convincingly that the novel hypoxia-regulated root agravitropism acts antagonistically to ERFVII function. Observed root bending in erfVII was much greater than in wild-type plants and is therefore an ERFVII-independent lowoxygen regulated process, requiring the existence of another oxygen-sensing mechanism that may target auxin transport. Each laboratory studied different aspects of the hypoxia response, which may provide clues as to the nature of other lowoxygen associated sensing mechanisms. One element that springs to mind is the accumulation of the phytohormone ethylene that always occurs in submerged plants (used in Giuntoli et al.) due to reduced gas movement into the surrounding liquid, compared to seedlings in hypoxic gaseous environment (used in Eysholdt-Derzsó and Sauter). Testing whether the different phenotypes are reciprocated under the different physiological conditions could address this important point. Results from both laboratories, assaying different processes, leads to the potentially thrilling conclusion that the $\mathrm{N}$-end rule regulated ERFVII oxygen sensing mechanism is not alone in plants.

This neatly leads to the final of the three papers, in which Wang et al. discuss the possibility of ion channels as novel oxygen sensors in plants. The paper is part of a special focus issue of Plant and Cell Physiology, dedicated to sensors and sensing in plants. The authors argue that the reported timescales of stabilization of ERFVIIs in response to hypoxia and submergence are far too slow to account for observed changes in response to these stresses, including membrane depolarization, production of reactive oxygen species (ROS) and changes in transporter activity, indicating that non- N-end rule/ERFVII oxygen sensors may exist. They suggest that ERFVIIs should be considered as 'transducers' of the response, rather than true 'sensors'. This point remains moot as experiments to directly assess the rapidity of ERFVII stabilization in response to low oxygen have not been reported. Wang et al. used non- $\mathrm{N}$-end rule associated oxygen-sensing protein domains from archaebacteria to humans and ion channels from mammalian oxygen sensors in a bioinformatics approach to 
find $A$. thaliana proteins that contain similar sequences. This successfully identified a number of proteins that can be tested as potential new oxygen sensors in plants.

In addition to these proteins it is also possible that the oxygen-sensing capacity of the $\mathrm{N}$-end rule pathway works on non-ERFVII substrates. There are approximately 250 proteins in A. thaliana that, like ERFVIIs, have Met-Cys- at their amino terminus, and could therefore act as sensors if an amino-terminal $(\mathrm{N})$-degron is revealed following Met cleavage. Sensors could also be derived from proteins cleaved internally by non-processive endopeptidases to reveal amino-terminal Cys as part of an $\mathrm{N}$-degron (identification would require specific proteomics approaches that identify the amino-terminal amino acid, or clever genetic screens, perhaps using the newly revealed root agravitropic phenotype).

Although it is clear that ERFVIIs direct the brute force of the plant response to hypoxia by $\mathrm{N}$-end rule regulated oxygen sensing13, novel sensors may be involved in specific responses to low oxygen perceived in the environment or internally during normal growth and development. The data provided in these three papers indicate the exciting possibility of the presence of undiscovered oxygen-sensing capacity in plants, and provide tools with which to direct the search.

Michael J. Holdsworth School of Biosciences, University of Nottingham, Loughborough LE12 5RD, UK.e-mail: Michael.Holdsworth@nottingham.ac.uk

Published online: 2 October 2017 DOI: 10.1038/s41477-017-0031-7

\section{References}

1. The Impact of Natural Hazards and Disasters on Agriculture and Food Security and Nutrition (Food and Agriculture Organization of the United Nations, 2015). 2. Kelliher, T. \& Walbot, V. Science 337, 345-348 (2012). 3. Abbas, M. et al. Curr. Biol. 25, 1483-1488 (2015). 4. Bailey-Serres, J. \& Voesenek, L. Annu. Rev. Plant Biol. 59, 313-339 (2008). 5. Giuntoli, B. et al. Plant Cell Environ. https://doi.org/10.1111/ pce.13037 (2017). 6. EysholdtDerzso, E. \& Sauter, M. Plant Physiol. 175, 412-423 (2017). 7. Wang, F., Chen, Z.-H. \& Shabala, S. Plant Cell Physiol. 58, 1126-1142 (2017). 8. Gibbs, D. J. et al. Nature 479, 415418 (2011). 9. Licausi, F. et al. Nature 479, 419-422 (2011). 10. Gibbs, D. J. et al. Mol. Cell 53, 369-379 (2014). 11. Weits, D. A. et al. Nature Commun. 5, 3425 (2014). 12. Kosmacz, M. et al. Plant Cell Environ. 38, 1094-1103 (2015). 13. van Dongen, J. T. \& Licausi, F. Annu. Rev. Plant Biol. 66, 345-367 (2015). 14. Gibbs, D. J. et al. Plant Physiol. 169, 23-31 (2015). 15. Papdi, C. et al. Plant J. 82, 772-784 (2015).

Competing interests The authors declare no competing financial interests. 